\title{
Surgically Assisted Rapid Palatal Expansion and Mini-screw Assisted Rapid Palatal Expansion - A review
}

\author{
Dr. Vijay Kumar Vaghela \\ Orthodontist, Myra Dental Clinic, Ahmedabad, India \\ Email: v.k.vaghela@gmail.com
}

\section{Abstract}

Research summary: This review was undertaken to compare the effects of surgically assisted rapid palatal expansion (SARPE) and mini-screw assisted rapid palatal expansion (MARPE). Discrepancies in the transverse relation between maxillary and mandibular dentition can result in the development of posterior cross bite. For the correction of posterior cross bite, maxillary expansion is frequently undertaken. The conventional approach for maxillary expansion includes expansion screw being anchored to the maxillary teeth and is known and rapid palatal expansion (RPE) appliance. However, RPE can only be applied in growing individuals and is not as effective in late adolescents and adults. In such patients, surgically assisted rapid palatal expansion (SARPE) technique is used to expand the maxillary arch. As this procedure involves surgery, it deters many patients from choosing SARPE as a treatment option. Recently a nonsurgical technique of expansion of maxillary arch has been introduced that can be applied to late adolescents and adults using palatal mini-screws. This technique is known as mini-screw assisted rapid palatal expansion (MARPE). In this review, the studies in the current literature regarding the SARPE and MARPE expansion techniques as compared to conventional RPE were included. It was observed that both SARPE and MARPE are effective methods for achieving maxillary expansion. With both techniques being effective, the advantage of MARPE over SARPE is the non-surgical approach which leads to higher patient acceptance and lower rate of complications.

Keywords: Orthodontics, surgically assisted rapid palatal expansion, SARPE, MARPE Miniscrew assisted rapid palatal expansion, Surgical Orthodontics. 


\section{Introduction}

Discrepancy in the transverse dimension between maxillary arch and mandibular arch can lead to the development of posterior crossbite either unilateral, or bilateral. ${ }^{1,2}$ The treatment of such a transverse discrepancy was reported first by Emerson Angell in 1860, where he described how the palatal expansion appliance can be used to apply transverse forces and open the mid palatal suture. ${ }^{3}$ After almost a century, Andrew Hass popularized the palatal expansion approach in the united states and reported that expansion appliances lead to increased arch-width of maxilla. ${ }^{4}$

Rapid palatal expansion (RPE) has been used in orthodontics commonly to correct such malocclusion nowadays. Rapid Palatal Expansion (RPE) is known by different names such as tooth-anchored expansion appliance, rapid maxillary expansion (RME). It is a useful appliance for the correction of posterior in young patients. But in patients over the age of 15 years, the RPE appliance can lead to decreased skeletal changes and higher dental effects such as buccal dental tipping of the molars, root resorption, and alveolar bone-loss. ${ }^{5}$ This happens due to the maturation of the mid-palatal suture in older age. Thus, in order to overcome the resistance of the mid-palatal suture, surgical approaches have been undertaken to increase the maxillary transverse dimension. Such approaches consist of segmental LeFort-I osteotomy and Surgically Assisted Rapid Palatal Expansion (SARPE). ${ }^{6,7}$

Segmental LeFort-I osteotomy has been applied for correction of transverse discrepancy of 6-7 $\mathrm{mm}$ in maxilla. However, it has been reported to be unstable and a high tendency of relapse. ${ }^{6}$ Surgically assisted rapid palatal expansion (SARPE) was introduced by Brown in 1938 and has become a popular option since then for the treatment of transverse discrepancy in adults. The main objective with SARPE approach is to reduce the resistance rendered by midpalatal suture and lateral maxillary sutures so that a higher amount of skeletal expansion can be achieved. After performing the surgical procedure for the separation of the sutures, maxillary expansion is commonly achieved with the help of tooth-anchored expansion appliances for SARPE. This leads to similar side effects as conventional RPE such as dental tipping, root resorption, and periodontal issues. In addition, the surgical procedure deters many patients from choosing SARPE as a treatment option.

Because of these disadvantages, a non-surgical procedure that can lead to predictable opening of the mid-palatal suture was desired. 
Thus, Mini-screw assisted rapid palatal expansion (MARPE) appliances were introduced to the orthodontic armamentarium. MARPE appliances are a relatively newer option for the correction of posterior crossbite and can be used to achieve higher amount of skeletal expansion of maxilla than conventional RPE. ${ }^{8,9,10}$ Different devices using mini-implants and maxillary expansion have been reported in the literature. ${ }^{11}$ This review will describe the changes achieved with conventional RPE, SARPE, and MARPE.

\section{Materials and methods}

A thorough search of the literature was performed for the evaluation of studies investigating the effects of RPE, SARPE, and MARPE. The common parameters assessed for the evaluation of dental expansion were interpremolar width, intermolar width, and the width of nasal floor after MARPE and SARPE. Three dimensional radiographs such as cone-beam computed tomography scan (CBCT) is advantageous as compared to two dimensional radiographs such as lateral cephalometric radiograph and panoramic radiograph. Most of recent studies utilized Cone-beam computed tomography for the evaluation of the effects of the expansion appliances.

\section{Results}

The distance between the mesiobuccal cusp tips of maxillary first molar has been used to define the maxillary intermolar width. ${ }^{13,14}$ In some studies, the distance between the central fossa of maxillary first molars has been used to define the maxillary intermolar width. ${ }^{8}$

Regardless of the method used for measurement, the difference between the pre-treatment and post-expansion are used to calculate the amount of the expansion.

Nada et al. performed a study in 2012 on patients who underwent surgical procedure for expansion of maxilla and observed that there was an increase of $5.64 \mathrm{~mm}+/-2.9 \mathrm{~mm}$ in the intermolar width after expansion. ${ }^{13}$ Kayaler et al. in 2016 found that the width at maxillary molar region increased more than the width at maxillary premolar region when expansion was undertaken with mini-screw assisted rapid palatal expansion. ${ }^{14}$ In the studies on MARPE by Park et al and Lin et al, the maxillary intermolar width increased by $5.4 \mathrm{~mm}$ and $5.63 \mathrm{~mm}$ respectively. ${ }^{15,16}$ But these studies only showed the short-term effects of expansion and long term effects of expansion were not analyzed in these studies. 
Recently, a study conducted by Mehta et al. described the long term effects of mini-screw assisted rapid palatal expansion and conventional tooth-anchored rapid palatal expansion. ${ }^{8}$ This study was conducted on a sample from randomized controlled clinical trial on three groups : i) Mini-screw assisted rapid palatal expansion, ii) conventional rapid palatal expansion, and iii) controls. ${ }^{8}$ They showed in the study that the maxillary intermolar width increased by $4.51 \mathrm{~mm}$ (95\% CI: 3.46 to 5.55 ) in the short-term and 5.24 (95\% CI: 3.98 to $6.5 \mathrm{~mm}$ ) in the long term in mini-screw assisted rapid palatal expansion. In the conventional rapid palatal expansion group, the maxillary intermolar width was found to be increased by $6.07 \mathrm{~mm}$ (95\% CI: $5.09 \mathrm{~mm}$ to 7.05 $\mathrm{mm})$ in the short term and $4.2 \mathrm{~mm}(95 \% \mathrm{CI}: 3.13 \mathrm{~mm}$ to $5.27 \mathrm{~mm})$ in the long term. In the control group, the maxillary intermolar width increased by $0.02 \mathrm{~mm}$ (95\% CI: -0.16 to $0.21 \mathrm{~mm}$ ) and $3.64 \mathrm{~mm}$ (95\% CI: 2.52 to $4.75 \mathrm{~mm}){ }^{8}$

An important point to consider for the success of the MARPE appliance is the success rate of palatal mini-implants. As compared to buccal mini-implants, buccal shelf mini-implants, interradicular mini-implants, palatal mini-implants have the highest success rates. In addition, the expansion appliances are cemented either on teeth (RPE) or on mini-implants (MARPE).

\section{Discussion}

In this review, studies analyzing the effects of SARPE and MARPE were evaluated to find out which technique and appliance is better for orthodontic expansion. ${ }^{8,13-16}$ In this review, only the studies using Cone-beam Computed Tomography (CBCT) were included for the evaluation of the effects of expansion appliances. This is because CBCT can provide an accurate picture of expansion by comparing the parameters before and after expansion. a more sophisticated radiographic examination could be performed with micro-computed tomography (micro-CT). However, even though micro-CT can be used in animal studies, it cannot be utilized in human studies because of the amount of radiation and thus, the next closest technique to micro-CT for head and neck is CBCT, which has a lower radiation and a high accuracy. The research conducted on expansion appliances in earlier times used two-dimensional radiographs which can cause errors in assessment because of the errors of magnification in such radiographs. Furthermore, with two-dimensional radiographs, the head positioning errors can cause inaccuracies in measurement of maxillary and mandibular structures. ${ }^{17}$ 
With the introduction of mini-screws in the orthodontic field, the orthodontic biomechanics have undergone a significant evolution. ${ }^{18}$ Thus, in the newer studies, an increased emphasis has been put on researching the effects of mini-screws and orthodontic expansion appliances. ${ }^{19}$ It is vital to understand the anatomy and morphology of midpalatal suture and the effect of age on midpalatal suture. It has been reported that the midpalatal suture undergoes fusion with age. ${ }^{20}$ Thus, with the fusion of midpalatal suture at higher age, it is imperative to understand that performing conventional rapid palatal expansion would not lead to opening of midpalatal suture. Because of this, higher dental effects would be observed in such patients in late adolescents or older. This would lead to undesirable results such as dental tipping of molars and root resorption. In such cases, a surgical option such as SARPE or a non-surgical option such as MARPE would be beneficial in opening the midpalatal suture and decrease the incidence of dental side effects. ${ }^{13}$ Also, it has been reported that MARPE does not cause side-effects to the mandibular condyle and glenoid fossa relationship. ${ }^{11}$

In our review, all the studies showed that both treatment strategies SARPE and MARPE are effective in achieving maxillary expansion. These findings stand to reason that if both strategies are effective, why not opt for a non-surgical strategy such as MARPE in lieu of SARPE as it saves the patient the trauma and complication of undergoing a surgical procedure. Furthermore, in the short-term multiple studies showed that both SARPE MARPE led to a significant increase in the intermolar width.

However, it is necessary to examine the long-term findings as it would allow us to observe whether the short term changes with expansion are stable in the long term. The only study with long term follow up after MARPE as compared to RPE and controls was the one performed by Mehta et al. ${ }^{8}$ In this study, the authors found that the type of expansion with conventional RPE was triangular. However, with MARPE, a parallel expansion was observed. ${ }^{8}$ This is because as MARPE obtains anchorage from mini-implants and not from teeth. ${ }^{8}$ All the studies showed that within 3-6 months after expansion, the maxillary intermolar width increased with MARPE and SARPE. ${ }^{8,13-16}$ Mehta et al. had the longest follow up period of 2.5 years and showed that MARPE led to an increase in the maxillary intermolar width even after 2.5 years indicating that the results with MARPE are stable in the long term. ${ }^{8}$ 
Even with SARPE technique, the expansion can be undertaken with conventional tooth-anchored rapid palatal expansion appliance or mini-screw assisted rapid palatal expansion appliance. ${ }^{13}$ After SARPE, mini-screw assisted rapid palatal expansion appliance has been shown to cause parallel expansion and minimal side effects as opposed to conventional tooth-borne expansion appliance. However, the clinician's skills and experience with mini-implants and MARPE appliance play an important role in the success of the appliance. Each case should be evaluated separately to identify if MARPE appliance would be required or not.

The landscape of orthodontics is continually changing and mini-implants have definitely brought change in the way orthodontics is practiced. With the help of artificial imaging, in the future the evaluation of the radiographs could be automated for enhanced accuracy and decreased subjectivity. ${ }^{21}$ The future studies should focus on long term effects of expansion appliances and incorporating artificial intelligence can be incorporated for the evaluation of the outcomes of different orthodontic expansion appliances.

\section{Conclusion}

Maxillary intermolar width increased predictably with both surgically assisted rapid palatal expansion (SARPE) and mini-screw assisted rapid palatal expansion (MARPE) procedures. SARPE procedure involves surgical intervention of the maxilla whereas MARPE can be used as a non-surgical alternative to SARPE for the expansion of maxillary arch.

\section{References}

1. Harrison, J. E., \& Ashby, D. (2000). Orthodontic treatment for posterior crossbites. The Cochrane database of systematic reviews, (2), CD000979.

2. Ramires, T., Maia, R. A., \& Barone, J. R. (2008). Nasal cavity changes and the respiratory standard after maxillary expansion. Brazilian journal of otorhinolaryngology, 74(5), 763-769.

3. Angell, E. C. (1860). Treatment of irregularities of the permanent or adult teeth. Dental Cosmos 1: 540-544. 
4. Hass A. J. (1961). Rapid expansion of the maxillary dental arch and nasal cavity by opening the midpalatal suture. Angle Orthod 31 (2): 73-90.

5. Lu, J., Gong, F. F., \& Shen, G. (2006). Shanghai kou qiang yi xue = Shanghai journal of stomatology, 15(4), 363-366.

6. Starch-Jensen, T., \& Blæhr, T. L. (2016). Transverse Expansion and Stability after Segmental Le Fort I Osteotomy versus Surgically Assisted Rapid Maxillary Expansion: a Systematic Review. Journal of oral \& maxillofacial research, 7(4), e1.

7. Brown GVI. The surgery of oral and facial diseases and malformations: their diagnosis and treatment including plastic surgical reconstruction. London: Lea and Febiger; 1938.

8. Mehta, S., Wang, D., Kuo, C. L., Mu, J., Vich, M. L., Allareddy, V., Tadinada, A., \& Yadav, S. (2021). Long-term effects of mini-screw-assisted rapid palatal expansion on airway. The Angle orthodontist, 91(2), 195-205.

9. Lee, K. J., Park, Y. C., Park, J. Y., \& Hwang, W. S. (2010). Miniscrew-assisted nonsurgical palatal expansion before orthognathic surgery for a patient with severe mandibular prognathism. American journal of orthodontics and dentofacial orthopedics : official publication of the American Association of Orthodontists, its constituent societies, and the American Board of Orthodontics, 137(6), 830-839.

10. Park, J. J., Park, Y. C., Lee, K. J., Cha, J. Y., Tahk, J. H., \& Choi, Y. J. (2017). Skeletal and dentoalveolar changes after miniscrew-assisted rapid palatal expansion in young adults: A cone-beam computed tomography study. Korean journal of orthodontics, 47(2), 77-86.

11. Mehta, S., Chen, P. J., Vich, M. L., Upadhyay, M., Tadinada, A., \& Yadav, S. (2021). Bone-anchored versus tooth-anchored expansion appliances: Long-term effects on the condyle-fossa relationship. Journal of the World federation of orthodontists, S22124438(21)00031-X. Advance online publication. https://doi.org/10.1016/j.ejwf.2021.07.001.

12. Berco, M., Rigali, P. H., Jr, Miner, R. M., DeLuca, S., Anderson, N. K., \& Will, L. A. (2009). Accuracy and reliability of linear cephalometric measurements from cone-beam 
computed tomography scans of a dry human skull. American journal of orthodontics and dentofacial orthopedics : official publication of the American Association of Orthodontists, its constituent societies, and the American Board of Orthodontics, 136(1), 17.e1-18.

13. Nada, R. M., Fudalej, P. S., Maal, T. J., Bergé, S. J., Mostafa, Y. A., \& Kuijpers-Jagtman, A. M. (2012). Three-dimensional prospective evaluation of tooth-borne and bone-borne surgically assisted rapid maxillary expansion. Journal of cranio-maxillo-facial surgery : official publication of the European Association for Cranio-Maxillo-Facial Surgery, 40(8), 757-762.

14. Kayalar, E., Schauseil, M., Kuvat, S. V., Emekli, U., \& Firatlı, S. (2016). Comparison of tooth-borne and hybrid devices in surgically assisted rapid maxillary expansion: A randomized clinical cone-beam computed tomography study. Journal of cranio-maxillofacial surgery : official publication of the European Association for Cranio-MaxilloFacial Surgery, 44(3), 285-293.

15. Nojima, L. I., Nojima, M., Cunha, A., Guss, N. O., \& Sant'Anna, E. F. (2018). Miniimplant selection protocol applied to MARPE. Dental press journal of orthodontics, 23(5), 93-101.

16. Lim, H. M., Park, Y. C., Lee, K. J., Kim, K. H., \& Choi, Y. J. (2017). Stability of dental, alveolar, and skeletal changes after miniscrew-assisted rapid palatal expansion. Korean journal of orthodontics, 47(5), 313-322.

17. Mehta, S., Dresner, R., Gandhi, V., Chen, P. J., Allareddy, V., Kuo, C. L., Mu, J., \& Yadav, S. (2020). Effect of positional errors on the accuracy of cervical vertebrae maturation assessment using CBCT and lateral cephalograms. Journal of the World federation of orthodontists, 9(4), 146-154.

18. Kim, S. H., Hwang, Y. S., Ferreira, A., \& Chung, K. R. (2009). Analysis of temporary skeletal anchorage devices used for en-masse retraction: a preliminary study. American journal of orthodontics and dentofacial orthopedics : official publication of the American Association of Orthodontists, its constituent societies, and the American Board of Orthodontics, 136(2), 268-276. 
19. Dzingle, J., Mehta, S., Chen, P. J., \& Yadav, S. (2020). Correction of Unilateral Posterior Crossbite with U-MARPE. Turkish journal of orthodontics, 33(3), 192-196.

20. Colonna, A., Cenedese, S., Sartorato, F., Spedicato, G. A., Siciliani, G., \& Lombardo, L. (2021). Association of the mid-palatal suture morphology to the age and to its density: A CBCT retrospective comparative observational study. International orthodontics, 19(2), $235-242$.

21. Mehta, S., Suhail, Y., Nelson, J., \& Upadhyay M. (2021). Artificial Intelligence for radiographic image analysis. Semin Orthod. 27:109-120

Copyright (C) 2021 Dr. Vijay Kumar Vaghela, AJRSP. This is an open-access article distributed under the terms of the Creative Commons Attribution License (CC BY NC).

Doi: doi.org/10.52132/Ajrsp.e.2021.302 